\title{
BHSF Palliative Care: COVID-19 Response
}

Ana Viamonte Ros, MD, MPH

Director of Bioethics \& Palliative Care

Brenda Daniels, MD

Symptom Management \& Palliative Medicine

\section{Nivia Ruiz, MD}

Symptom Management \& Palliative Medicine

Mayra Villalba, MSN, CMSRN

Palliative Care Clinical Educator
No one is ready for a pandemic but thanks to our wonderful teams throughout BHSF, our service was able to adapt to the "new normal" and meet the needs of communities and patients. Our team members strive to keep patients, families and themselves safe and Tele-Medicine has had a crucial part in allowing us to keep seeing patients regardless of their COVID diagnosis. Our teams have put in an incredible amount of work to ensure that patients we can't see physically still feel connected to their loved ones and involved in their care by facilitating phone conferences and zoom meetings.

During this period of unpredictability, we have disseminated symptom management protocols, algorithms and conversation guides to our referring partners and providers throughout the system to empower them and support the palliative care needs of their patients. These supportive tools provide sample medication strategies, goal oriented conversational phrases to establish a plan of care and address the importance of an Advance Directive, all which can be found on http://intranet.bhssf.org/en/ departments-and-directories/PalliativeCare/ Pages/COVID-19.aspxl.

In further preparation efforts, the Palliative Care teams have been in communication with the Pharmacy departments to ensure adequate supply of medication for palliation of symptoms. Palliative Care leadership has also developed a Phased response plan based on patient acuity, hospital capacity as well as availability of resources to ensure the most efficient use of our teams while prioritizing the conservation of PPE.
While COVID has presented many challenges, an opportunity presented itself to better support and manage the needs of our providers and patients in the form of an after-hours Palliative Care service manned by our Palliative Care physicians and our APRNs. Although this service was originally intended to operate during the COVID-19 pandemic, we have decided to adopt this service permanently. Operators receive bi-monthly schedules allowing ease of use from any facility. For nurses and other providers taking care of patients with active Palliative Care consults we have also included our direct contact information for physicians and APRNs at each respective site.

In order to best serve our communities, bedside staff, patients and families we have also collaborated with the South Miami Hospital Magnet Project Director and the 2 Tower nursing unit with education, tools and resources to care for and communicate with our patients and families facing end of life circumstances related and unrelated to COVID-19. The goal is to empower nurses and increase their confidence with these delicate and difficult conversations while ensuring adequate symptom management for patients.

Palliative Care Medical Director, Dr. Ana Viamonte Ros and Mayra Villalba, Palliative Care clinical educator, have also been working on wellbeing physician and APP resources titled "Be Well" to address the emotional and psychosocial needs of those taking care of our patients and families. This resource can be found on https://bewell.baptisthealth.net/. 